\title{
The medium to long-term role of renewable energy sources in climate change mitigation in Portugal
}

\author{
Sofia Simões ${ }^{1, *}$, Júlia Seixas ${ }^{1}$, Patrícia Fortes ${ }^{1}$, Luís Dias ${ }^{1}$, João Gouveia ${ }^{1}$, Bárbara Maurício ${ }^{1}$ \\ ${ }^{1}$ CENSE, Departamento de Ciências e Engenharia do Ambiente, Faculdade de Ciências e Tecnologia, \\ Universidade Nova de Lisboa, Caparica, Portugal \\ *Corresponding author. Tel: +351 212948354, Fax: +351 212948354,E-mail: sgcs@fct.unl.pt
}

\begin{abstract}
Portuguese policy-makers have adopted ambitious targets for RES promotion until 2020, but there are no national targets for the medium to long -term (2050) and it is not clear to what extent which RES can contribute to CC mitigation. This paper aims to assess the contribution of RES for the CC mitigation in Portugal until 2050, under cost-effectiveness criteria. The TIMES_PT linear optimization bottom-up technology model was used to generate six scenarios to 2050 combining GHG emission caps, levels of socio-economic growth and share of RES electricity. In order to meet the 2050 energy demand, the share of RES in primary energy consumption increases 4 to 6 times from 2005 and in final energy grows from 15\% in 2005 to 56-59\% in 2050 . RES were found to be cost-effective even without a GHG cap. Regarding CC mitigation the high RES shares in final energy correspond to less 49-74\% GHG emissions in 2050 compared to a baseline without cap. The role of renewable electricity is determinant to mitigate CC especially due to hydro and onshore wind. Other important deployments of RES technologies are solar water heating and heat pumps in buildings, biomass use for process heat in industry and biodiesel in transport.
\end{abstract}

Keywords: Climate change mitigation, Renewable energy, Energy modeling, Portugal.

\section{Introduction}

Renewable energy sources (RES) play a key-role in climate change (CC) mitigation. Moreover, RES have added benefits of reducing external energy dependency and fostering economic development. Acknowledging this, Portugal has been pointed worldwide as a success case for RES deployment (IEA, 2009, NYT, 2010). National CC \& energy policymakers have adopted ambitious targets for RES promotion until 2020. The National Energy Strategy for 2020 (Cabinet Resolution n. ${ }^{\circ}$ 29/2010 of April 15) defines the following main objectives: i) reduce the external energy dependency to $74 \%$ (it was $87 \%$ in 2008); ii) ensure compliance of commitments within EU climate change policies, allowing that in $202060 \%$ of generated electricity is renewable based (RES-E) and 31\% of final energy consumption is from RES (respectively 50\% RES-E in September 2010 and 20\% in 2005), and iii) achieving a reduction of $20 \%$ final energy consumption in the terms of the Energy-Climate policy package. The Portuguese National Action Plan (PNAER) within the Directive 2009/28/EC sets even more ambitious policies \& measures (P\&M) that will allow reaching 70\% RES-E in 2020 and 10\% biofuels in transport (update on PNAER by the Decree-Law $n^{\circ} 117 / 2010$ of October 25). Other P\&M are in place to promote RES heating and cooling and end-use energy efficiency, namely through the National Energy Efficiency Action Plan (RCM 80/2008).

Although there is high policy focus on medium-term RES promotion (2020) there are no national targets for the medium to long-term (2050). Likewise there are no quantitative estimates on avoided GHG emissions due to RES promotion, both in medium and long-term. Furthermore, there is no information on which RES (e.g. solar or waves) and which RES technologies (e.g. PV panels or biomass boilers) are the most cost-effective for Portugal. This is highly relevant to support national policy making, particularly regarding the design of incentives to promote the most cost-effective RES. This paper aims to assess the contribution of RES for the reduction of GHG emissions in Portugal until 2050 looking into detail into which technologies are most cost-effective. 


\section{Methodology}

To assess the role of RES in CC mitigation in Portugal up to 2050, we used the TIMES_PT model to generate six scenarios combining different assumptions as presented in Table 1.

Table 1. GHG and RES scenarios for Portugal up to 2050

\begin{tabular}{cccc}
\hline Scenario & GHG cap & Economic Growth $^{\text {a) }}$ & Minimum fossil electricity \\
\hline C & None & Conservative & $30 \%$ of total electricity \\
F & None & Fenix & $30 \%$ of total electricity \\
$-50 \mathrm{C}$ & $-50 \%$ in $2050 / 1990$ & Conservative & $30 \%$ of total electricity \\
$-50 \mathrm{~F}$ & $-50 \%$ in $2050 / 1990$ & Fenix & $30 \%$ of total electricity \\
Cefre & None & Conservative & None \\
Fefre & None & Fenix & None \\
\hline
\end{tabular}

a) Two socio-economic scenarios were developed for Portugal as briefly outlined below.

To assess RES contribution to CC mitigation, we consider a $\mathbf{G H G}^{\mathbf{1}}$ emission cap in the -50C and -50F scenarios starting from 2015 with $+27 \%$ of the 1990 (the Kyoto target for 20102012 extended to 2015) and linearly more stringent until -50\% of 1990 for combustion and productive processes GHG emissions in 2050. (A trend line was then generated from the 2015 to the 2050 cap to obtain intermediate emission caps for every 5 years. The $-50 \%$ cap is quite severe as it roughly leads to per capita emissions of 2.04 t CO2e in 2050 whereas in 2008 Portugal had 7.4 t CO2e. The per capita EU 15 average in 2008 was 10.1 tCO2e according to EEA data.

Regarding economic growth and demand for energy services, two contrasting socioeconomic scenarios were used: Conservative and Fenix. The Conservative scenario follows the current economic and demographic trends (1\% GDP annual growth rate and population decrease); whereas the Fenix scenario has more optimistic economic and population evolution forecasts (2 to 2.26\% GDP annual growth rates and more 12\% inhabitants in 2050 compared to 2005). These scenarios were used to generate two demand projections for materials and energy services such as residential lighting or cement which are inputs of the TIMES_PT model. More information on the demand projections and can be found on Seixas et al. (2009).

Finally, in four of the six studied scenarios (C, F, -50C and -50F) we assumed a conservative requirement to assure the reliability of the power system translated as a minimum of $\mathbf{3 0 \%}$ of total generated electricity is produced by centralized fossil plants from 2015 to 2050. In the Cefre and Fefre scenarios we removed this constraint and the system is free to adopt as much RES-E as needed according to cost-effectiveness criteria. Such approach could be associated with ensuring security of supply via expanded transmission capacity and increased electricity trade. In this paper however, we do not deal with electricity trade. We assumed that the net electricity imports are nil from 2025 onwards following the Portuguese transmission system operator expectations. If assumed otherwise the entire configuration of the electricity system would alter depending on how much electricity could be exported. However, at the moment there are absolutely no expectations on amounts of electricity traded after 2025 and any scenarios would by highly uncertain and out of the scope of this paper. Thus we have focused instead on the cost-effective assessment of maximum potential of national renewable

\footnotetext{
${ }^{1}$ This paper solely refers to energy related GHG emissions, i.e. from fuel combustion activities, fugitive emissions from oil, natural gas and other sources and from major industrial processes. These were approximately $81 \%$ of 2005 national emissions.
} 
resources for the national CC mitigation considering nil electricity imports after 2025.

All these assumptions were inputted into the linear optimization bottom-up technology TIMES_PT ${ }^{2}$ model which represents the Portuguese energy system from 2005 to 2050. The TIMES_PT is an implementation of the TIMES family of models developed by ETSAP of IEA which has been implemented at global, regional or national level (ETSAP, 2008), namely for the whole of UE (Pan European Times model from the NEEDS project) or for the countries Spain (Labriet, et al., 2010), Belgium (Proost, et al., 2009) or Germany (Blesl, et al., 2007), among other EU countries. It considers both the supply and demand sides and disaggregates the energy demand sectors. The model is supported by a detailed database, which includes the technical and economical characteristics of the existing and future energy technologies and present and future sources of primary energy supply and their maximum technical and economic potentials (e.g. maximum available biomass or area for solar panels). TIMES_PT finds the optimum combination of energy supply and demand technologies to satisfy the demand with the lowest possible total costs. More information on the details of the model can be found in Simões et al. (2008) and more details on the technology and primary energy assumptions in Seixas et al. (2009). The learning curves for RES-E solar and wave technologies are from the IEA (IEA, 2010, IEA, 2008) which were validated by national stakeholders. Wind RES-E technologies learning curves were supplied by national experts of the National Energy and Geology Research Institute (LNEG, 2010).

Other exogenous assumptions are very briefly outlined: 1) 8\% discount rate for centralized electricity generation, buses and trains; $12 \%$ for commercial, industry, decentralized electricity generation, CHP and freight transport; and $17.5 \%$ for residential, cars and motorcycles. 2) maximum of $5000 \mathrm{Gg} \mathrm{CO} 2$ carbon capture and storage potential were assumed as available since there is no data at the moment available for Portugal. More information on CCS cost data can be found at Simões et al. (2008); 3) no nuclear due to current policies and the purpose of this work focusing the role of RES; 4) new coal power plants without CCS not allowed due to climate policy; 5) RES targets, subsidies or feed-in tariffs not considered; 6) cost of oil barrel of 100 USD\$ 2008 for the year 2020, 115 in 2030 and 145 in 2050.

\section{Results}

\subsection{RES in primary energy consumption}

In order to meet the 2050 energy demand, the share of RES in primary energy consumption can increase to two to three times the 2005 values in the scenarios without GHG emission cap (Figure 1) which shows the cost-effectiveness of RES. To meet the CO2 caps (-50C and -50F) RES can further increase to 4 to 6 times the 2005 values. The most competitive RES in all scenarios are wind and hydro which achieve its maximum potential in 2050. Solar, national biomass and, to a lesser scale, geothermal are also competitive but only if a GHG cap is in place. Removing the $30 \%$ fossil electricity requirement does not lead to significant changes in RES in 2020. However, in 2050 the higher RES-E share leads to higher consumption of solar especially in the Fefre scenario, where it achieves its maximum potential.

The increase in RES allows decreasing the external energy dependency from $87 \%$ in 2008 to $70-77 \%$ in 2020 and to $58-72 \%$ in 2050 . The lowest values are not obtained due to the GHG

\footnotetext{
2 The Portuguese model development was undertaken within the EU FP7 research project NEEDS (www.needs-project.org). The NEEDS RS2a research team is responsible for the model structure. The authors are responsible for some structural changes, the base-year and new technologies information and for calibration and validation of the national model.
} 
cap but instead due to 100\% RES-E. If a backup of 30\% fossil electricity is removed imports of natural gas for centralized CCGT plants can be reduced already in 2020. In any case, in 2050 a new energy import paradigm appears; instead of being dependent on imported fossil fuels the energy system will import biomass, particularly biofuels for transports.

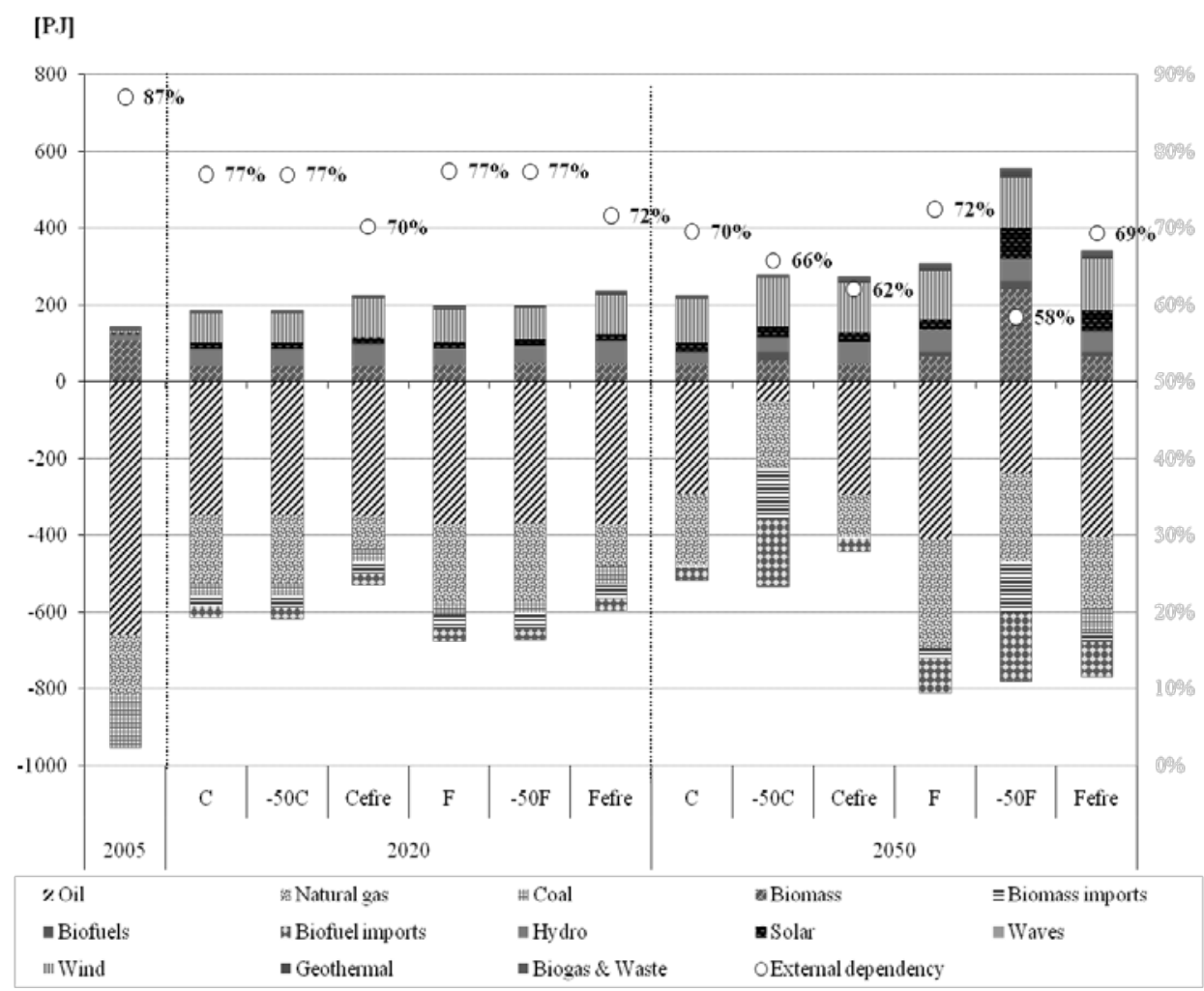

Fig. 1. Primary energy consumption in the studied scenarios, $\%$ of external energy dependency and \% of RES (in the top rectangle). The lower values for 2020 are due to increase of refinery exports, decommissioning of a major coal power plant and slow recovery from 2010-2015 economic crisis.

\subsection{RES in electricity generation}

Until 2020 the electricity sector profile will be similar to 2009 since it will rely in recent investments both on RES (wind and hydro) and on new gas CCGT. Globally, approximately $58 \%$ of total electricity in 2020 is RES-E, in all scenarios with minimum $30 \%$ fossil electricity. In 2050 the GHG cap has a significant effect in RES-E technologies profile only in the $-50 \mathrm{~F}$ scenario since there is a higher overall demand for electricity (107.16 TWh, in comparison with $84.03 \mathrm{TWh}$ in F). In -50C wind and hydro are sufficient to meet the demand. The system will firstly use all available hydro and onshore wind resources and in $-50 \mathrm{~F}$ this is followed by centralised PV, biomass and biogas CHP, and both geothermal steam turbines and hot dry rock systems. These technologies are practically negligible in 2050 in C, F, and 50C as the demand for RES-E is not high enough also due to the requirement for minimum $30 \%$ electricity from centralised fossil fuel. Without this requirement, in Cefre and Fefre, already in 2020 at least $78 \%$ of electricity will be RES-E and hydro and wind potentials will be achieved (9.7 and 6.5 GW, respectively). In 2050 74-89\% electricity is RES and in Fefre large PV plants achieve the maximum potential $(9.33 \mathrm{GW})$ and appears $0.50 \mathrm{GW}$ of wind offshore. Both in 2020 and 2050 the gas CCGT plants will not work due to higher fuel and O\&M costs.

In Cefre and Fefre there is a lower demand for electricity than in the other scenarios due to the higher contribution of efficient equipments and appliances in buildings, district heating in 
commercial and biomass and insulation in the residential. This means that the $30 \%$ fossil electricity requirement hampers energy efficiency and RES use in final energy.

\subsection{RES in final energy consumption}

Concerning final energy consumption (FEC) in 2020, no significant changes in the energy profile are expected, even with the cap, although RES share increases from $15 \%$ of total FEC in 2005 to $30-35 \%$. In the long term (2050) it is clear that the increase in electricity is a major strategy to mitigate CC as there are endogenous energy resources used to generate RES-E, especially wind and hydro, as mentioned before. In 2050, the FEC in the F scenario is almost $70 \%$ higher than in the $\mathrm{C}$ leading to new technologies to meet the cap, such as $\mathrm{H} 2$ for transports. The share of RES in FEC in 2050 varies from 31-36\% in scenarios without the GHG cap to 56-59\% with the cap (Table 2). The RES share grows more due to the GHG cap in the transports (both in $\mathrm{C}$ and $\mathrm{F}$ ) and industry (only in the -50F scenario) sectors.

Table 2. RES contribution in final energy consumption for the six scenarios

\begin{tabular}{cccccccc}
\hline Sector/Scenario & 2005 & \multicolumn{7}{c}{2050} \\
\cline { 3 - 8 }$[\mathrm{PJ}]$ & & $\mathrm{C}$ & $-50 \mathrm{C}$ & Cefre & $\mathrm{F}$ & $-50 \mathrm{~F}$ & Fefre \\
\hline RES Electricity & 32 & 159 & 188 & 203 & 221 & 300 & 252 \\
RES Heath \& cold & 96 & 69 & 68 & 69 & 91 & 206 & 93 \\
Residential & 50 & 34 & 32 & 34 & 39 & 38 & 39 \\
Commercial & 0 & 11 & 8 & 10 & 14 & 13 & 13 \\
Industry & 45 & 25 & 27 & 25 & 39 & 155 & 41 \\
RES in Transport & 0 & 36 & 245 & 36 & 98 & 200 & 98 \\
Final Renewable Energy (a) & 128 & 263 & 501 & 307 & 410 & 707 & 442 \\
Total Final Energy (b) & 826 & 863 & $\mathbf{8 4 6}$ & 860 & 1225 & 1253 & 1224 \\
\% Renewables (a/b) & $\mathbf{1 5}$ & $\mathbf{3 1}$ & $\mathbf{5 9}$ & $\mathbf{3 6}$ & $\mathbf{3 3}$ & $\mathbf{5 6}$ & $\mathbf{3 6}$ \\
\hline
\end{tabular}

Other relevant uses of RES are solar for water and space heating in buildings, which in all scenarios, regardless of GHG cap and RES-E restrictions; achieve its maximum potential already in 2020. In 2050 with the GHG cap, solar panels are also cost-effective to generate heat for industry and the potential is also achieved. The role of biomass is reduced in buildings as electricity, solar thermal and heat pumps become more appealing. On the other hand, biomass will become more cost-effective in CHP to generate heat for industry. In transports the share of biofuels is expected to increase above $10 \%$ in 2020 in all scenarios and in 2050 up to $60-40 \%$ due to the GHG cap. Other impacts of the GHG cap in 2050 in the transport sector are to create room and need for electric vehicles and for $\mathrm{H} 2$ freight trucks.

\section{Discussion}

We found that RES technologies are highly cost-effective in the Portuguese energy system even without any CO2 cap (36-38\% of PEC and 31-33\% of FEC in 2050). If an ambitious CC mitigation cap is in place, the contribution of RES is even higher to 65-72\% of PEC and 59$56 \%$ of FEC in 2050. If the layout of the power sector does not require centralised fossil plants, for example by ensuring security of supply via expanded transmission capacity, RES contribute with $41-44 \%$ of PEC and 36\% of FEC in 2050. So, a cap on GHG emissions has a larger impact in RES contribution than a reconfiguration of the power system. Although RES play a fundamental role in CC mitigation in Portugal it should be noted that it is not possible to reduce external energy dependency below 77\% in 2020 and below 50\% in 2050. Further reductions are only possible with stronger efforts on energy efficiency, which were not in the 
scope of this paper.

Regarding RES technologies, hydro and wind power can achieve the maximum technical and economic potential in Portugal in the medium run (2020) and contribute significantly to generated electricity. To some extent, this already occurs as in 2009 wind and hydro ensured $34 \%$ of total generated electricity. Until 2050 they can generate $60-80 \%$ of total electricity, respectively if a GHG cap is in place or if no fossil electricity backups are required. On the other hand, electricity generation technologies from solar are still in an early-phase and need extra incentives to become competitive before 2050. Nonetheless, policy support to solar technologies should be considered from a R\&D perspective anticipating future technology costs reductions since Portugal already has know-how in this area and some national companies manufacture components. Electricity generation from waves and offshore wind technologies are competitive from 2035 onwards only if Portugal adopts an aggressive GHG cap or no centralised fossil backup is needed. In these conditions and considering the existing national $R \& D$ capacities and wind parts supply chain, these two technology groups should be considered by policy makers as a priority.

Besides RES electricity, solar (both for water and space heating) is highly competitive already in the medium term (2020) even without any GHG emission cap. Heat pumps are also extremely competitive but only if a cap is in place. On the other hand electric vehicles are only cost-effective in 2050 if a cap is in place and the technology evolves to supply longdistance mobility as existing cars do. Otherwise, biofuels are a cheaper alternative.

Finally, the results presented have the following main caveats: 1) learning curve for energy technologies with high uncertainty, especially for the least mature technologies; 2) high uncertainty of profile of electricity trade within the Iberian electricity market; 3) high uncertainty on the availability of endogenous and imported biomass and biofuels. Moreover, the TIMES_PT is a partial equilibrium model and thus does not model economic interactions outside the energy sector and does not consider in detail demand curves and non-rational aspects that condition investment in new technologies. All of these caveats reflect real life uncertainties which policy makers have to deal with especially when thinking of long-term policies. An approach to try to handle uncertainty is to perform sensitivity analysis which the authors did for the RES electricity technologies learning curve (solar, wind offshore and waves) and for available biofuels and biomass. For electricity trade this was no done due to lack of any indication of plausible scenarios and involved amount of work considering the scope of the paper, as mentioned in section 2. It was found that assumptions on the technology learning rate affect the share of the different RES-E technologies in the energy system but the total share of RES-E is not altered. Variations on the amounts and prices of available biomass significantly affect RES potential for CC mitigation in Portugal, as biomass and biofuels are preferable to RES-E in the industry and transport sectors, since they are more cost-effective. However, it is not in the scope of this paper to discuss and assess uncertainty in detail and thus it is not possible here to present and discuss in detail the performed sensitivity analysis, but only to draw attention to the limitations of the results, which serve to illustrate that in Portugal RES are very effective for CC mitigation goals.

\section{Conclusions}

This paper's objective is to assess the contribution of RES for CC mitigation in Portugal until 2050 looking into detail into which technologies are most cost-effective. We have found that the RES share in final energy consumption can increase from 15\% in 2005 to 31-33\% in 2050 in a baseline scenario without an emission cap. This illustrates that RES are cost-effective 
regardless of the goal of CC mitigation, especially in the electricity generation sector (mostly hydropower and wind onshore technologies). To meet the GHG cap of-50\% in 2050 this share can further increase to 56-59\% of total final energy consumption. This represents a growth of more than $200 \%$ of 2005 values. Although the increase of energy efficiency is an alternative cost-effective strategy to CC mitigation, the GDP energy intensity in 2050 is only less 32$40 \%$ of 2005 values. This seems to suggest that RES can contribute more significantly to the emission targets than energy efficiency improvements.

Regarding GHG emission reduction, a49-74\% emission reduction is achieved in 2050 for the $-50 \%$ cap compared to the baseline. Electricity generation is the most relevant sector for abatement. This sector can be responsible for up to $98 \%$ of all abatement in 2050 if the constraint of a minimum of $30 \%$ total generated electricity is produced by centralized fossil plants is not present. In this situation all electricity will be renewable. In the scenarios where this minimum fossil electricity is required the electricity sector is not completely renewable and the transport sector is the most important sector for total GHG abatement (up to $57 \%$ of total GHG emission reduction in 2050 compared to baseline). In both sectors RES are the main reason for emission abatement, both hydropower and onshore wind technologies, followed to a lesser extent by solar PV and geothermal electricity generation technologies, and biofuels for individual cars and freight trucks.

\section{References}

[1] IEA. Energy Policies of IEA Countries - Portugal 2009 Review. OECD / International Energy Agency. Paris, France, 2010, pp. 131-146.

[2] J. Seixas, S. Simões, P. Fortes, L. Dias, J. Gouveia, B. Alves, B. Maurício, [New Energy Technologies: Road Map Portugal 2050, D3: Competitiveness Assessment of New Energy Technologies], Novas Tecnologias Energéticas: Road Map Portugal 2050, D3: Análise da Competitividade das Novas Tecnologias Energéticas, Portuguese Innovation Fund for Renewable Energy of the Ministry of Economy, 2010, pp. 1-88.

[3] NYT. Portugal Gives Itself a Clean-Energy Makeover, The New York Times, August 10 ${ }^{\text {th }}$, 2010.pp. A1. Available at:

[http://www.nytimes.com/2010/08/10/science/earth/10portugal.html?_r=2\&ref=globalhome]

[4] ETSAP - International Energy Agency Implementing Agreement for a Programme of Energy Technology Systems Analysis, Global Energy Systems and Common Analysis Final Report of Annex X (2005-2008), Ed. G. Goldstein, G. Tosato, ETSAP, 2008, pp. 21 $-77$.

[5] S. Simões, J. Cleto, P. Fortes, J. Seixas, G. Huppes, Cost of energy and environmental policy in Portuguese $\mathrm{CO}_{2}$ abatement - scenario analysis to 2020. Energy Policy, 2008, Vol. 36, Issue 9, pp. 3598 - 3611.

[6] M. Labriet, H. Cabal, Y. Lechon, G. Giannakidis, A. Kanudia, The implementation of the EU renewable directive in Spain. Strategies and challenges. Energy Policy, 2010, Vol. 38, Issue 5, pp. 2272 - 2281.

[7] S. Proost, E. Delhaye, W. Nijs, D. van Regemorter, Will a radical transport pricing reform jeopardize the ambitious EU climate change objectives? Energy Policy, 2009, Vol. 37, Issue 10, pp. 3863 - 3871.

[8] M. Blesl, A. Das, U. Fahl, U. Remme, Role of energy efficiency standards in reducing CO2 emissions in Germany: An assessment with TIMES. Energy Policy, 2007, Vol. 35, 
Issue 2, pp. 772 - 785.

[9] IEA, Technology Roadmap - Solar Photovoltaic Energy, OECD/International Energy Agency. Paris, France, 2010, pp. 7 - 9.

[10] IEA, Technology Roadmap - Concentrating Solar Power, OECD/International Energy Agency. Paris, France, 2010, pp. 27 - 28.

[11]IEA, Energy Technology Perspectives, OECD/International Energy Agency. Paris, France, 2008, pp. 400.

[12]LNEG, 2010. Personnel communication from Eng. Ana Estanqueiro from LNEG Unit of Solar, Wind and Ocean Energy. June 16, 2010. 